\title{
A User-Friendly Extrapolation Method for Oscillatory Infinite Integrals
}

\author{
By Avram Sidi
}

\begin{abstract}
In a recent publication [4] the author developed an extrapolation method, the $W$-transformation, for the accurate computation of convergent oscillatory infinite integrals. In yet another publication [6] this method was shown to be applicable to divergent oscillatory infinite integrals that are defined in the sense of summability. The application of the $W$-transformation involves some asymptotic analysis of the integrand as the variable of integration tends to infinity. In the present work the $W$-transformation is modified so as to keep this asymptotic analysis to a minimum, involving only the phase of oscillations. This modified version, which turns out to be as efficient as the original $W$-transformation, can also be applied to convergent or divergent oscillatory infinite integrals other than those dealt with in [4] and [6]. The convergence properties of the modified transformation are analyzed in detail for the integrals of [4] and [6], and numerical examples are provided.
\end{abstract}

1. Introduction. In [4] the author developed an extrapolation method, the $W$ transformation, by which a large class of convergent infinite oscillatory integrals can be computed very efficiently. The approach of [4] was later extended to divergent infinite oscillatory integrals in [6], and it was shown that the $W$-transformation, with no modifications, can be applied to such integrals with the same efficiency. The use of the $W$-transformation involves some asymptotic analysis of the integrand as the variable of integration tends to infinity. The purpose of the present work is to modify the $W$-transformation so that this asymptotic analysis is kept to a minimum.

For future use and reference, we now summarize some of the notation and results of [4] and [6].

Definition 1.1. We say that a function $\alpha(x)$ belongs to the set $A^{(\gamma)}$ if it is infinitely differentiable for all $x>a \geq 0$, for some $a$, and if, as $x \rightarrow \infty$, it has a Poincaré type asymptotic expansion of the form

$$
\alpha(x) \sim x^{\gamma} \sum_{i=0}^{\infty} \alpha_{i} / x^{i}
$$

and if all its derivatives, as $x \rightarrow \infty$, have Poincaré type asymptotic expansions which are obtained by differentiating the right-hand side of (1.1) term by term.

Definition 1.2. We say that a function $f(x)$ belongs to $\mathbf{B}$ if it can be expressed in the form

$$
f(x)=\sum_{j=1}^{r} u_{j}\left(\theta_{j}(x)\right) \exp \left(\phi_{j}(x)\right) h_{j}(x)
$$

Received September 15, 1987.

1980 Mathematics Subject Classification (1985 Revision). Primary 40A10, 41A25, 41A60, 65B05, $65 \mathrm{D} 30$ 
where

(1) $u_{j}(z)$ is $e^{i z}$ or $e^{-i z}$ or any linear combination of these (like $\cos z$ or $\sin z$ ).

(2) $\theta_{j}(x)$ and $\phi_{j}(x)$ are real and $\theta_{j} \in A^{(m)}$ and $\phi_{j} \in A^{(k)}$ for all $j$, where $m$ is a positive integer and $k$ is a nonnegative integer. If, as $x \rightarrow \infty$,

$$
\theta_{j}(x) \sim \sum_{s=0}^{\infty} \theta_{j s} x^{m-s} \quad \text { and } \quad \phi_{j}(x) \sim \sum_{s=0}^{\infty} \phi_{j s} x^{k-s}
$$

and we set

$$
\bar{\theta}_{j}(x)=\sum_{s=0}^{m-1} \theta_{j s} x^{m-s} \quad \text { and } \quad \bar{\phi}_{j}(x)=\sum_{s=0}^{k-1} \phi_{j s} x^{k-s},
$$

then we assume that

$$
\bar{\theta}_{j}(x) \equiv \bar{\theta}_{p}(x) \quad \text { and } \quad \bar{\phi}_{j}(x) \equiv \bar{\phi}_{p}(x) \quad \text { whenever } j \neq p \text {. }
$$

We will denote $\bar{\theta}_{j}(x)=\bar{\theta}(x)$ and $\bar{\phi}_{j}(x)=\bar{\phi}(x)$. Note that $\bar{\theta}(x)$ and $\bar{\phi}(x)$ are the polynomial parts of $\theta_{j}(x)$ and $\phi_{j}(x)$, respectively, and $\Delta_{j}(x)=\theta_{j}(x)-\bar{\theta}(x)$ and $\Lambda_{j}(x)=\phi_{j}(x)-\bar{\phi}(x)$ are both in $A^{(0)}$. We also assume that if $k \geq 1$, then $\lim _{x \rightarrow \infty} \phi_{j}(x)=-\infty$, i.e., $\phi_{j 0}<0$. Obviously, when $k=0, \bar{\phi}_{j}(x) \equiv 0, j=1, \ldots, r$. Without loss of generality we will assume that $\lim _{x \rightarrow \infty} \theta_{j}(x)=\infty$, i.e., $\theta_{j 0}>0$.

(3) $h_{j} \in A^{\left(\gamma_{j}\right)}$ for some arbitrary $\gamma_{j}$ such that $\gamma_{j}-\gamma_{p}$ are integers for all $j$ and $p$ when $r>1$. We will denote $\gamma=\max \left\{\gamma_{1}, \ldots, \gamma_{r}\right\}$.

The set $\mathbf{B}$ is the union of the two mutually exclusive subsets that were denoted $\mathbf{B}_{c}$ and $\mathbf{B}_{d}$ in [6]. $\mathbf{B}_{c}$ is the set of functions in $\mathbf{B}$ that are integrable at infinity, i.e., functions $f(x)$ for which either $k \geq 1$ or $k=0$ and $\gamma<m-1$. $\mathbf{B}_{d}$ on the other hand is the set of functions in $\mathbf{B}$ that are not integrable at infinity, i.e., those functions $f(x)$ for which $k=0$ and $\gamma \geq m-1$. It was shown in [6] that integrals of functions in $\mathbf{B}_{d}$ exist in the sense of Abel summability, and that the $W$-transformation that was developed for integrals of functions in $\mathbf{B}_{c}$ can be applied to integrals of functions in $\mathbf{B}_{d}$ without any changes.

Let $f \in \mathbf{B}$ and be of the form described in Definition 1.2 with the notation therein. Let $a \geq 0$, and define

$$
I[f]=\int_{a}^{\infty} f(t) d t \quad \text { and } \quad F(x)=\int_{a}^{x} f(t) d t
$$

where $I[f]$ is to be taken as $\int_{a}^{\infty} f(t) d t$ in the Abel summability sense when $f \in \mathbf{B}_{d}$. Then, as is shown in [4] and [6],

$$
I[f]=F(x)+x^{\sigma+\gamma} \exp (\bar{\phi}(x))\left[\cos (\bar{\theta}(x)) b_{1}(x)+\sin (\bar{\theta}(x)) b_{2}(x)\right],
$$

where $b_{1}, b_{2} \in A^{(0)}$ and

$$
\sigma=\min \{-m+1,-k+1\}=1-\max \{m, k\} .
$$

Now let $x_{0}$ be the first zero of $\sin (\bar{\theta}(x))$ that is greater than $a$. This means that $x_{0}$ is a root of the polynomial equation $\bar{\theta}(x)=q \pi$ for some integer $q$. Then determine $x_{l}$ to be the largest positive root of the polynomial equation $\bar{\theta}(x)=(q+l) \pi$, $l=1,2, \ldots$, i.e., $x_{l}, l=0,1, \ldots$, are ultimately consecutive zeros of the function 
$\sin (\bar{\theta}(x))$. This guarantees $x_{0}<x_{1}<x_{2} \cdots$. Obviously, $\lim _{l \rightarrow \infty} x_{l}=\infty$. The $W$-transformation is then defined by the solution of the linear system of equations

$$
W_{n}^{(j)}=F\left(x_{l}\right)+\psi\left(x_{l}\right) \sum_{i=0}^{n} \beta_{i} / x_{l}^{i}, \quad j \leq l \leq j+n+1,
$$

in which $W_{n}^{(j)}$ and the $\beta_{i}$ are unknown, $W_{n}^{(j)}$ is the approximation to $I[f]$, and

$$
\psi\left(x_{l}\right)=(-1)^{l} x_{l}^{\sigma+\gamma} \exp \left(\bar{\phi}\left(x_{l}\right)\right), \quad l=0,1, \ldots
$$

The equations (1.9) and (1.10) are obtained as follows: Let $x=x_{l}$ in (1.7) so that the term $\sin (\bar{\theta}(x)) b_{2}(x)$ disappears; the result is

$$
I[f]=F\left(x_{l}\right)+(-1)^{l} x_{l}^{\sigma+\gamma} \exp \left(\bar{\phi}\left(x_{l}\right)\right) b_{1}\left(x_{l}\right),
$$

where the constant multiplicative factor $(-1)^{q}$ has been absorbed into $b_{1}\left(x_{l}\right)$. Recall that $b_{1} \in A^{(0)}$, so that $b_{1}(x) \sim \sum_{i=0}^{\infty} b_{1 i} / x^{i}$ as $x \rightarrow \infty$. Truncate this expansion at the term $b_{1 n} / x^{n}$ and replace $b_{1}\left(x_{l}\right)$ in (1.11) by this truncated series. Now replace $I[f]$ by $W_{n}^{(j)}$ and $b_{1 i}$ by $\beta_{i}$, treat these as unknowns, and let $l=j, j+1, \ldots, j+n+1$ so as to get (1.9).

The solution of the system for $W_{n}^{(j)}$ can be achieved very efficiently by the $W$ algorithm of [5]; here are the steps of this algorithm: Set

$$
M_{-1}^{(s)}=F\left(x_{s}\right) / \psi\left(x_{s}\right), \quad N_{-1}^{(s)}=1 / \psi\left(x_{s}\right), \quad s=0,1, \ldots,
$$

and compute, for $s=0,1, \ldots, p=0,1, \ldots$,

$$
\begin{aligned}
M_{p}^{(s)} & =\left(M_{p-1}^{(s)}-M_{p-1}^{(s+1)}\right) /\left(x_{s}^{-1}-x_{s+p+1}^{-1}\right), \\
N_{p}^{(s)} & =\left(N_{p-1}^{(s)}-N_{p-1}^{(s+1)}\right) /\left(x_{s}^{-1}-x_{s+p+1}^{-1}\right), \\
W_{p}^{(s)} & =M_{p}^{(s)} / N_{p}^{(s)} .
\end{aligned}
$$

Now, as is shown in [3] and [5], $W_{n}^{(j)}$ is of the form

$$
W_{n}^{(j)}=\sum_{i=0}^{n+1} \gamma_{n, i}^{(j)} F\left(x_{j+i}\right)
$$

where

$$
\sum_{i=0}^{n+1} \gamma_{n, i}^{(j)}=1 \text { for all } j \text { and } n
$$

and

$$
\gamma_{n, i}^{(j)}>0, \quad 0 \leq i \leq n+1, \quad \text { for all } j \text { and } n,
$$

the latter being a direct consequence of

$$
\psi\left(x_{l}\right) \psi\left(x_{l+1}\right)<0, \quad l \geq 0,
$$

which in turn holds by (1.10). Actually, (1.17) is necessary and sufficient for (1.16) to hold. Now (1.10), (1.11), and (1.14)--(1.16) enable us to prove that

$$
I[f]-W_{n}^{(j)}=O\left[x_{j}^{\sigma+\gamma-n-1} \exp \left(\bar{\phi}\left(x_{j}\right)\right)\right] \text { as } j \rightarrow \infty \quad \text { (Process I) }
$$


and

$$
I[f]-W_{n}^{(j)}=o\left(n^{-\mu}\right) \quad \text { as } n \rightarrow \infty, \text { all } \mu>0 \quad \text { (Process II). }
$$

More will be said in Section 3 concerning (1.14)-(1.19).

As can be seen from (1.7)-(1.10), the $W$-transformation involves the asymptotic analysis of the integrand $f(x)$ for $x \rightarrow \infty$, some of which is quantitative in nature. In particular, $\psi\left(x_{l}\right)$ requires knowledge of $\bar{\theta}(x), \bar{\phi}(x)$, and $\gamma$. In the next section we show how the $W$-transformation can be modified so as to keep the asymptotic analysis of $f(x)$ to a minimum (involving $\bar{\theta}(x)$ only), without affecting its numerical efficiency very much. In fact, in all of our numerical experiments we have observed that both the original and the modified $W$-transformations have comparable accuracy when applied to integrals of functions in B. In Section 3 we analyze the convergence properties of the modified $W$-transformation for these integrals and show that (1.18) and (1.19) are still valid. By employing an approach different from the one used in [3], [4], and [6], we actually prove a significantly improved version of (1.18), which is valid for the (original) $W$-transformation as well as the modified one. The main results for the modified $W$-transformation are given in Theorem 3.1 (Process I), and in Lemma 3.8 and Theorem 3.2 (Process II). Finally, the modified $W$-transformation seems to produce very accurate results also for integrals of functions $f(x)$ that oscillate as those in $\mathbf{B}$ but are not in $\mathbf{B}$ themselves. This is a surprising observation for which we have no explanation at present. More details on this are given in the next section.

Before proceeding to the next section, we mention that $x_{l}, l=0,1, \ldots$, can be taken to be consecutive zeros of the function $\cos (\bar{\theta}(x))$ instead of $\sin (\bar{\theta}(x))$, everything else, including the convergence results of Section 3 , remaining the same.

2. The Modified $W$-Transformation. The modification that we propose to make in the $W$-transformation consists of replacing (1.10) by

$$
\psi\left(x_{l}\right)=\int_{x_{l}}^{x_{l+1}} f(t) d t=F\left(x_{l+1}\right)-F\left(x_{l}\right), \quad l=0,1,2, \ldots
$$

$W_{n}^{(j)}$ is again defined through the linear system of equations in (1.9) and hence can be computed recursively by the $W$-algorithm as described in (1.12) and (1.13).

As is shown in Lemma 3.3 of the present work, for $l$ sufficiently large, the $\psi\left(x_{l}\right)$ alternate in sign.

Since $F\left(x_{l}\right)=\sum_{i=-1}^{l-1} \psi\left(x_{i}\right)$, where we have set $\psi\left(x_{-1}\right)=\int_{a}^{x_{0}} f(t) d t$, we see that the only quantities required for the determination of $W_{n}^{(j)}$ in the modified $W$-transformation are the finite integrals $\psi\left(x_{i}\right),-1 \leq i \leq j+n+1$. Thus, the only information needed beyond $f(t)$ is the set of first few $x_{l}$, which in turn is obtained from the polynomial $\bar{\theta}(x)$. That is to say, in order to apply the modified $W$-transformation, we need only consider the dorninant polynomial part of the phase of oscillations and need not concern ourselves with the modulating factors such as $h_{j}(x) \exp \left(\phi_{j}(x)\right)$. This offers a significant advantage, as it suggests that we could at least attempt to apply the modified $W$-transformation to all oscillatory infinite integrals whose integrands are of the form

$$
f(x)=\sum_{j=1}^{r} u_{j}\left(\theta_{j}(x)\right) H_{j}(x)
$$


where $u_{j}(z)$ and $\theta_{j}(x)$ are exactly as described in Definition 1.2 , and the $H_{j}(x)$ are arbitrary functions that do not oscillate for $x \rightarrow \infty$. We might even consider very slow oscillations (compared to $\exp ( \pm i \bar{\theta}(x)))$ in $H_{j}(x)$ such as $\exp ( \pm i R(x)$ ), where as $x \rightarrow \infty, R(x) \sim c(\log x)^{k}, k>0$. Indeed, numerical results suggest that the modified $W$-transformation is as efficient on such integrals as on those with integrands in $\mathbf{B}$.

Since the modified $W$-transformation is ultimately based on the $\psi\left(x_{l}\right)$ only, it can be viewed as a method in which one first integrates $f(t)$ between the zeros of $\sin (\bar{\theta}(x))$ (or $\cos (\bar{\theta}(x)))$ to obtain the integrals $\psi\left(x_{l}\right)$, and then accelerates the convergence of the (ultimately alternating) infinite series $\sum_{i=-1}^{\infty} \psi\left(x_{i}\right)$, which converges to $I[f]$ in case $f \in \mathbf{B}_{c}$. In this sense, the modified $W$-transformation is akin to a method first proposed by Longman in [1], in which one integrates $f(t)$ between its consecutive zeros $y_{1}<y_{2}<\cdots, y_{1}>a=y_{0}$, to obtain the integrals $v_{i}=\int_{y_{i}}^{y_{i+1}} f(t) d t, i=0,1, \ldots$, and then accelerates the convergence of the alternating infinite series $\sum_{i=0}^{\infty} v_{i}$ by a sequence transformation, e.g., the Euler transformation.

We now apply the modified $W$-transformation to some infinite integrals with integrands in $\mathbf{B}$ or as in (2.2). In all cases, the approximations presented are $W_{n}^{(0)}$, $n=0,1,2, \ldots$ The computation of the $\psi\left(x_{l}\right)$ is performed accurately by a loworder Gaussian quadrature formula, although other numerical quadrature methods can be employed. As is seen from the numerical values of $F\left(x_{i}\right)$ given in Tables $2.1-2.5$, the $\psi\left(x_{l}\right)$ are alternating in sign, as expected.

The computations reported in this section were carried out in double-precision arithmetic on an IBM-370 computer.

Example 2.1. $I_{p}=\int_{0}^{\infty} x^{2 p} J_{0}(x) d x$.

Using the original $W$-transformation, this integral was computed in [6] for $p=1$ and $p=2$. As mentioned there, since $J_{0}(x)=\eta_{1}(x) \cos x+\eta_{2}(x) \sin x$, where $\eta_{1}, \eta_{2} \in A^{(-1 / 2)}$, the integrand $f(x)=x^{2 p} J_{0}(x)$ is in $\mathbf{B}_{d}$ whenever $p \geq 1 / 4$, so that $I_{p}$ is defined in the sense of Abel summability. Of course, $\bar{\theta}(x)=x$, hence, $x_{l}=(l+1) \pi, l=0,1, \ldots$ We again computed $I_{p}$ for $p=1$ and $p=2$ for which $I_{1}=-1$ and $I_{2}=9$. The results of the computation are given in Table 2.1.

Example 2.2. $\int_{0}^{\infty} e^{i \omega(x)} \omega(x) \omega^{\prime}(x) d x=e^{i \omega(0)}[-1+i \omega(0)]$.

Whenever $\omega(x)$ is real and is in $A^{(q)}$ for some positive integer $q$, the integrand is in $\mathbf{B}_{d}$. An integral of exactly this type was computed in [6] by the $W$-transformation. Actually, the integrand is in $\mathbf{B}_{d}$ also when $\omega(x)$ is complex and $\lim _{x \rightarrow \infty} \operatorname{Im} \omega(x)=$ $C$, a constant. When $\lim _{x \rightarrow \infty} \operatorname{Im} \omega(x)=+\infty$, however, it is in $\mathbf{B}_{c}$.

In this work we apply the modified $W$-transformation to the convergent integrals

$$
I_{s}=I\left[f_{s}\right]=\int_{0}^{\infty} f_{s}(t) d t, \quad s=1,2,3,
$$

where $f_{s}(x)=\operatorname{Re}\left[e^{i \omega_{s}(x)} \omega_{s}(x) \omega_{s}^{\prime}(x)\right]$, and

$$
\begin{array}{ll}
\omega_{1}(x)=\left(x^{2}+2 \sqrt{x^{2}+x+4}-4\right)+i \varepsilon\left((x+1)^{3}-1\right), & \varepsilon>0, \\
\omega_{2}(x)=\left((x+1)^{3}-1\right)+i \varepsilon\left(x^{2}+2 \sqrt{x^{2}+x+4}-4\right), & \varepsilon>0,
\end{array}
$$


TABLE 2.1

$F\left(x_{n}\right)$ and relative error in $W_{n}^{(0)}$ for the integrals $I_{1}=-1$ and

$I_{2}=9$ of Example 2.1, using the modified $W$-transformation.

\begin{tabular}{|r|r|l|r|l|}
\hline $\mathrm{n}$ & $F\left(x_{n}\right)$ for $I_{1}$ & $\begin{array}{l}\text { relative error in } \\
W_{n}^{(0)} \text { for } I_{1}\end{array}$ & $F\left(x_{n}\right)$ for $I_{2}$ & $\begin{array}{l}\text { relative error in } \\
W_{n}^{(0)} \text { for } I_{2}\end{array}$ \\
\hline & & & & \\
0 & $-0.776 \mathrm{D}+01$ & $0.865 \mathrm{D}+00$ & $-0.973 \mathrm{D}+02$ & $0.250 \mathrm{D}+01$ \\
1 & $0.128 \mathrm{D}+02$ & $0.371 \mathrm{D}-01$ & $0.824 \mathrm{D}+03$ & $0.540 \mathrm{D}+00$ \\
2 & $-0.233 \mathrm{D}+02$ & $0.171 \mathrm{D}-02$ & $-0.271 \mathrm{D}+04$ & $0.214 \mathrm{D}-02$ \\
3 & $0.309 \mathrm{D}+02$ & $0.101 \mathrm{D}-03$ & $0.654 \mathrm{D}+04$ & $0.428 \mathrm{D}-02$ \\
4 & $-0.437 \mathrm{D}+02$ & $0.443 \mathrm{D}-05$ & $-0.131 \mathrm{D}+05$ & $0.918 \mathrm{D}-03$ \\
5 & $0.535 \mathrm{D}+02$ & $0.657 \mathrm{D}-07$ & $0.234 \mathrm{D}+05$ & $0.478 \mathrm{D}-04$ \\
6 & $-0.681 \mathrm{D}+02$ & $0.615 \mathrm{D}-08$ & $-0.383 \mathrm{D}+05$ & $0.206 \mathrm{D}-05$ \\
7 & $0.796 \mathrm{D}+02$ & $0.470 \mathrm{D}-09$ & $0.590 \mathrm{D}+05$ & $0.388 \mathrm{D}-07$ \\
8 & $-0.959 \mathrm{D}+02$ & $0.744 \mathrm{D}-11$ & $-0.867 \mathrm{D}+05$ & $0.446 \mathrm{D}-10$ \\
9 & $0.109 \mathrm{D}+03$ & $0.912 \mathrm{D}-12$ & $0.123 \mathrm{D}+06$ & $0.473 \mathrm{D}-10$ \\
10 & $-0.127 \mathrm{D}+03$ & $0.143 \mathrm{D}-12$ & $-0.168 \mathrm{D}+06$ & $0.197 \mathrm{D}-11$ \\
11 & $0.141 \mathrm{D}+03$ & $0.742 \mathrm{D}-13$ & $0.224 \mathrm{D}+06$ & $0.754 \mathrm{D}-12$ \\
12 & $-0.160 \mathrm{D}+03$ & $0.895 \mathrm{D}-13$ & $-0.293 \mathrm{D}+06$ & $0.181 \mathrm{D}-11$ \\
13 & $0.176 \mathrm{D}+03$ & $0.104 \mathrm{D}-12$ & $0.375 \mathrm{D}+06$ & $0.270 \mathrm{D}-11$ \\
14 & $-0.197 \mathrm{D}+03$ & $0.118 \mathrm{D}-12$ & $-0.472 \mathrm{D}+06$ & $0.323 \mathrm{D}-11$ \\
15 & $0.213 \mathrm{D}+03$ & $0.128 \mathrm{D}-12$ & $0.587 \mathrm{D}+06$ & $0.317 \mathrm{D}-11$ \\
16 & $-0.235 \mathrm{D}+03$ & $0.135 \mathrm{D}-12$ & $-0.719 \mathrm{D}+06$ & $0.239 \mathrm{D}-11$ \\
17 & $0.253 \mathrm{D}+03$ & $0.135 \mathrm{D}-12$ & $0.872 \mathrm{D}+06$ & $0.120 \mathrm{D}-11$ \\
18 & $-0.276 \mathrm{D}+03$ & $0.123 \mathrm{D}-12$ & $-0.105 \mathrm{D}+07$ & $0.425 \mathrm{D}-12$ \\
19 & $0.295 \mathrm{D}+03$ & $0.968 \mathrm{D}-13$ & $0.125 \mathrm{D}+07$ & $0.122 \mathrm{D}-11$ \\
\hline
\end{tabular}

and

$$
\omega_{3}(x)=\omega_{1}(x)+\frac{1}{10} \log (1+x) .
$$

As mentioned above, $f_{s}(x), s=1,2$, are in $\mathbf{B}_{c}$, but $f_{3}(x)$ is not, although $\int_{0}^{\infty} f_{3}(t) d t$ converges. In fact, $f_{3}(x)$ is not even in $\mathbf{B}$. Now if $\varepsilon$ is chosen to be very close to zero, then the integrals $\int_{0}^{\infty} f_{s}(t) d t$ converge very slowly. Indeed, their convergence is not noticeable in practice.

We note that for $f_{1}(x)$ we have $m=2$ and $k=3$, while for $f_{2}(x), m=3$ and $k=2$. For $f_{1}(x), \bar{\theta}(x)=x^{2}+2 x$, so that $x_{l}=-1+\sqrt{1+(l+1) \pi}$, and for $f_{2}(x), \bar{\theta}(x)=(x+1)^{3}-1$, so that $x_{l}=-1+(1+(l+1) \pi)^{1 / 3}$. The treatment of $f_{3}(x)$ is identical to that of $f_{1}(x)$. The reason for this is that the dominant polynomial part of the phase of oscillations is $\bar{\theta}(x)=x^{2}+2 x$. We include the factors $\exp ( \pm i / 10 \log (1+x))$ in the amplitude, as they oscillate at a much slower rate than $\exp ( \pm i \bar{\theta}(x))$. The results of the computation for $\varepsilon=10^{-4}$ are given in Table 2.2.

Example 2.3.

$$
\int_{0}^{\infty} \frac{\sin \alpha x}{x} \frac{\sinh \beta x}{\sinh \gamma x} d x=\arctan \left(\tan \frac{\beta \pi}{2 \gamma} \tanh \frac{\alpha \pi}{2 \gamma}\right) .
$$

For $0<\beta<\gamma$ this integral converges, but its integrand is not in $\mathbf{B}$. In fact, the integrand is of the form (2.2) with $\bar{\theta}(x)=\alpha x$. Therefore, we could apply the modified $W$-transformation with $x_{l}=(l+1) \pi / \alpha$. The results of the computation for $\alpha=1, \beta=0.1$, and $\gamma=0.2$ are given in Table 2.3 . 
TABLE 2.2

$F\left(x_{n}\right)$ and relative error in $W_{n}^{(0)}$ for the integrals $I_{s}=-1, s=1,2,3$, of Example 2.2, using the modified $W$-transformation.

\begin{tabular}{|c|c|c|c|c|c|c|}
\hline $\mathrm{n}$ & $F\left(x_{n}\right)$ for $I_{1}$ & $\begin{array}{l}\text { relative error in } \\
W_{n}^{(0)} \text { for } I_{1}\end{array}$ & $F\left(x_{n}\right)$ for $I_{2}$ & $\begin{array}{l}\text { relative error in } \\
W_{n}^{(0)} \text { for } I_{2}\end{array}$ & $F\left(x_{n}\right)$ for $I_{3}$ & $\begin{array}{l}\text { relative error in } \\
W_{n}^{(0)} \text { for } I_{3}\end{array}$ \\
\hline 0 & $-0.570 D+01$ & $0.618 D+00$ & $-0.145 \mathrm{D}-07$ & $0.163 \mathrm{D}-08$ & $-0.566 \mathrm{D}+01$ & $0.618 D+00$ \\
\hline 1 & $0.668 D+01$ & $0.391 \mathrm{D}-01$ & $-0.200 D+01$ & $0.535 \mathrm{D}-09$ & $0.681 \mathrm{D}+01$ & $0.389 \mathrm{D}-01$ \\
\hline 2 & $-0.113 D+02$ & $0.783 \mathrm{D}-02$ & $-0.552 \mathrm{D}-07$ & $0.127 \mathrm{D}-09$ & $-0.117 D+02$ & $0.753 \mathrm{D}-02$ \\
\hline 3 & $0.117 D+02$ & $0.287 \mathrm{D}-03$ & $-0.200 D+01$ & $0.245 \mathrm{D}-10$ & $0.124 D+02$ & $0.254 \mathrm{D}-03$ \\
\hline 4 & $-0.158 D+02$ & $0.578 \mathrm{D}-04$ & $-0.115 \mathrm{D}-06$ & $0.419 \mathrm{D}-11$ & $-0.169 D+02$ & $0.635 \mathrm{D}-0.4$ \\
\hline 5 & $0.158 D+02$ & $0.101 \mathrm{D}-04$ & $-0.200 D+01$ & $0.705 \mathrm{D} \cdot 12$ & $0.173 \mathrm{D}+02$ & $0.106 \mathrm{D}-04$ \\
\hline 6 & $-0.196 \mathrm{D}+02$ & $0.601 \mathrm{D}-06$ & $-0.189 \mathrm{D}-06$ & $0.870 \mathrm{D}-13$ & $-0.216 \mathrm{D}+02$ & $0.510 \mathrm{D}-06$ \\
\hline 7 & $0.193 D+02$ & $0.134 \mathrm{D}-07$ & $-0.200 D+01$ & $0.480 \mathrm{D}-13$ & $0.218 \mathrm{D}+02$ & $0.483 \mathrm{D}-07$ \\
\hline 8 & $-0.230 \mathrm{D}+02$ & 0.963 D-09 & $-0.275 \mathrm{D}-06$ & $0.298 D-13$ & $-0.259 D+02$ & $0.731 \mathrm{D}-08$ \\
\hline 9 & $0.225 \mathrm{D}+02$ & $0.147 \mathrm{D}-08$ & $-0.200 D+01$ & $0.331 D-13$ & $0.260 \mathrm{D}+02$ & 0.648 D-09 \\
\hline 10 & $-0.260 \mathrm{D}+02$ & $0.481 D-09$ & $-0.371 D-06$ & $0.264 \mathrm{D}-13$ & $-0.300 \mathrm{D}+02$ & $0.402 D \cdot 09$ \\
\hline 11 & $0.254 \mathrm{D}+02$ & $0.105 \mathrm{D}-09$ & $-0.200 \mathrm{D}+01$ & $0.107 \mathrm{D}-13$ & $0.300 \mathrm{D}+02$ & $0.103 \mathrm{D}-09$ \\
\hline 12 & $-0.287 D+02$ & $0.209 \mathrm{D}-10$ & $-0.477 \mathrm{D}-06$ & $0.145 \mathrm{D}-13$ & $-0.339 D+02$ & $0.222 \mathrm{D}-10$ \\
\hline 13 & $0.280 \mathrm{D}+02$ & $0.403 \mathrm{D}-11$ & $-0.200 D+01$ & $0.431 D-13$ & $0.338 \mathrm{D}+02$ & $0.456 \mathrm{D}-11$ \\
\hline 14 & $-0.312 \mathrm{D}+02$ & $0.843 D-12$ & $-0.591 \mathrm{D}-06$ & $0.653 \mathrm{D}-13$ & $-0.376 D+02$ & $0.968 \mathrm{D}-12$ \\
\hline 15 & $0.304 D+02$ & $0.102 \mathrm{D}-12$ & $-0.200 D+01$ & $0.738 \mathrm{D}-13$ & $0.374 \mathrm{D}+02$ & $0.136 \mathrm{D}-12$ \\
\hline 16 & $-0.336 \mathrm{D}+02$ & $0.810 \mathrm{D}-13$ & $-0.712 \mathrm{D}-06$ & $0.693 \mathrm{D}-13$ & $-0.412 D+02$ & 0.870 D. 13 \\
\hline 17 & $0.327 D+02$ & $0.413 \mathrm{D}-13$ & $-0.200 D+01$ & $0.601 \mathrm{D}-13$ & $0.409 D+02$ & $0.457 \mathrm{D}-13$ \\
\hline 18 & $-0.358 \mathrm{D}+02$ & $0.391 \mathrm{D}-13$ & $-0.841 \mathrm{D}-06$ & $0.547 \mathrm{D}-13$ & $-0.446 \mathrm{D}+02$ & $0.500 \mathrm{D}-13$ \\
\hline 19 & $0.348 D+02$ & $0.275 \mathrm{D}-13$ & $-0.200 D+01$ & $0.579 D-13$ & $0.442 D+01$ & $0.409 \mathrm{D}-13$ \\
\hline
\end{tabular}

\section{TABLE 2.3}

$F\left(x_{n}\right)$ and relative error in $W_{n}^{(0)}$ for the integral of Example 2.3 with $\alpha=1, \beta=0.1$, and $\gamma=0.2$, using the modified $W$-transformation.

Note that the exact value of the integral is 0.785398012695720765 and that $F\left(x_{20}\right)$ is correct to four decimal digits.

\begin{tabular}{|r|l|l|}
\hline $\mathrm{n}$ & $F\left(x_{n}\right)$ & $\begin{array}{c}\text { relative crror in } \\
W_{n}^{(0)}\end{array}$ \\
\hline 0 & $0.723 \mathrm{D}+00$ & $0.296 \mathrm{D}-02$ \\
1 & $0.820 \mathrm{D}+00$ & $0.788 \mathrm{D}-04$ \\
2 & $0.765 \mathrm{D}+00$ & $0.791 \mathrm{D}-05$ \\
3 & $0.798 \mathrm{D}+00$ & $0.501 \mathrm{D}-05$ \\
4 & $0.778 \mathrm{D}+00$ & $0.744 \mathrm{D}-06$ \\
5 & $0.790 \mathrm{D}+00$ & $0.275 \mathrm{D}-07$ \\
6 & $0.782 \mathrm{D}+00$ & $0.221 \mathrm{D}-07$ \\
7 & $0.787 \mathrm{D}+00$ & $0.215 \mathrm{D}-08$ \\
8 & $0.784 \mathrm{D}+00$ & $0.245 \mathrm{D}-09$ \\
9 & $0.786 \mathrm{D}+00$ & $0.840 \mathrm{D}-10$ \\
10 & $0.785 \mathrm{D}+00$ & $0.618 \mathrm{D}-11$ \\
11 & $0.786 \mathrm{D}+00$ & $0.114 \mathrm{D}-11$ \\
12 & $0.785 \mathrm{D}+00$ & $0.337 \mathrm{D}-12$ \\
13 & $0.786 \mathrm{D}+00$ & $0.231 \mathrm{D}-13$ \\
14 & $0.785 \mathrm{D}+00$ & $0.516 \mathrm{D}-14$ \\
15 & $0.785 \mathrm{D}+00$ & $0.221 \mathrm{D}-14$ \\
16 & $0.785 \mathrm{D}+00$ & $0.565 \mathrm{D}-15$ \\
17 & $0.785 \mathrm{D}+00$ & $0.442 \mathrm{D}-15$ \\
18 & $0.785 \mathrm{D}+00$ & $0.565 \mathrm{D}-15$ \\
19 & $0.785 \mathrm{D}+00$ & $0.618 \mathrm{D}-15$ \\
\hline
\end{tabular}


TABLE 2.4

$F\left(x_{n}\right)$ and relative error in $W_{n}^{(0)}$ for the integral of Example 2.4, using the modified $W$-transformation. The exact value of the integral is 0.421024438240708343 .

\begin{tabular}{|r|l|l|}
\hline $\mathrm{n}$ & $F\left(x_{n}\right)$ & $\begin{array}{l}\text { relative error in } \\
W_{n}^{(0)}\end{array}$ \\
\hline 0 & $-0.264 \mathrm{D}-01$ & $0.554 \mathrm{D}-03$ \\
1 & $0.850 \mathrm{D}+00$ & $0.550 \mathrm{D}-03$ \\
2 & $0.805 \mathrm{D}-02$ & $0.183 \mathrm{D}-03$ \\
3 & $0.820 \mathrm{D}+00$ & $0.307 \mathrm{D}-04$ \\
4 & $0.345 \mathrm{D}-01$ & $0.352 \mathrm{D}-05$ \\
5 & $0.797 \mathrm{D}+00$ & $0.388 \mathrm{D}-06$ \\
6 & $0.552 \mathrm{D}-01$ & $0.524 \mathrm{D}-07$ \\
7 & $0.778 \mathrm{D}+00$ & $0.879 \mathrm{D}-08$ \\
8 & $0.720 \mathrm{D}-01$ & $0.163 \mathrm{D}-08$ \\
9 & $0.763 \mathrm{D}+00$ & $0.319 \mathrm{D}-09$ \\
10 & $0.861 \mathrm{D}-01$ & $0.643 \mathrm{D}-10$ \\
11 & $0.750 \mathrm{D}+00$ & $0.134 \mathrm{D}-10$ \\
12 & $0.980 \mathrm{D}-01$ & $0.284 \mathrm{D}-11$ \\
13 & $0.739 \mathrm{D}+00$ & $0.619 \mathrm{D}-12$ \\
14 & $0.108 \mathrm{D}+00$ & $0.136 \mathrm{D}-12$ \\
15 & $0.729 \mathrm{D}+00$ & $0.313 \mathrm{D}-13$ \\
16 & $0.118 \mathrm{D}+00$ & $0.686 \mathrm{D}-14$ \\
17 & $0.720 \mathrm{D}+00$ & $0.181 \mathrm{D}-14$ \\
18 & $0.126 \mathrm{D}+\infty$ & $0.264 \mathrm{D}-15$ \\
19 & $0.713 \mathrm{D}+00$ & $0.198 \mathrm{D}-15$ \\
\hline
\end{tabular}

Example 2.4. $\int_{0}^{\infty} \frac{1}{2} \log \left(1+x^{2}\right) J_{1}(x) d x=K_{0}(1)$.

Because of $\log \left(1+x^{2}\right)=2 \log x+O(1)$ as $x \rightarrow \infty$, the integrand is not in $\mathbf{B}$. In view of $J_{1}(x)=\eta_{1}(x) \cos x+\eta_{2}(x) \sin x$, where $\eta_{1}, \eta_{2} \in A^{(-1 / 2)}$, however, it is of the form (2.2) with $\bar{\theta}(x)=x$. Thus, $x_{l}=(l+1) \pi$. The results of the computation are given in Table 2.4 .

Example 2.5. $\int_{0}^{\infty}\left(x^{3} J_{1}(x)-3 \cos (\omega(x)) \omega(x) \omega^{\prime}(x)\right) d x=0$, where $\omega(x)$ is real and contained in $A^{(q)}$ for some positive integer $q$ with $\omega(0)=0$.

We note that, even though both $f_{1}(x)=x^{3} J_{1}(x)$ and $f_{2}(x)=\cos (\omega(x)) \omega(x) \omega^{\prime}(x)$ are in $\mathbf{B}_{d}$, hence in $\mathbf{B}$, their linear combination is not. This is so because the $\gamma^{\prime} \mathbf{s}$ (see Definition 1.2) associated with $f_{1}(x)$ and $f_{2}(x)$ are $5 / 2$ and $2 q-1$, respectively, so that their difference is not an integer. Nevertheless, provided $\omega(x)=x+O(1)$ as $x \rightarrow \infty$, the integrand is of the form $(2.2)$ with $\bar{\theta}(x)=x$, so that $x_{l}=(l+1) \pi$. Table 2.5 gives the results of the computation for the case in which $\omega(x)=x+x /(10+x)$.

We note that the lack of improvement in the results towards the end of Tables $2.1,2.2$, and 2.5 is strictly due to the finite-precision arithmetic being used and the considerable difference between the orders of magnitude of the $F\left(x_{l}\right)$ and $I[f]$. A semiquantitative explanation of this is given below.

We recall that, if the computed value of $F\left(x_{l}\right)$ is $F\left(x_{l}\right)+\varepsilon_{l}$ and the $\gamma_{n, i}^{(j)}$ in (1.14) are assumed exact, then the computed value of $W_{n}^{(j)}$ is $W_{n}^{(j)}+E$, where $E=\sum_{i=0}^{n+1} \gamma_{n, i}^{(j)} \varepsilon_{j+i}$. Now, as is shown in Lemma 3.8 of this work, for fixed $j$, $\sum_{i=0}^{n+1}\left|\gamma_{n, i}^{(j)}\right| \rightarrow 1$ as $n \rightarrow \infty$. Thus, $|E| \leq(1+\eta) \max _{0 \leq i \leq n+1}\left|\varepsilon_{j+i}\right|$, where $\eta=o(1)$ 
TABLE 2.5

$F\left(x_{n}\right)$ and $W_{n}^{(0)}$ for the integral of Example 2.5. The exact value of the integral is zero.

\begin{tabular}{|r|r|r|}
\hline \multicolumn{1}{|c|}{$\mathrm{n}$} & $F\left(x_{n}\right)$ & $W_{n}^{(0)}$ \\
\hline 0 & $-0.852 \mathrm{D}+02$ & $-0.808 \mathrm{D}+01$ \\
1 & $0.210 \mathrm{D}+03$ & $0.197 \mathrm{D}+01$ \\
2 & $-0.403 \mathrm{D}+03$ & $-0.255 \mathrm{D}+00$ \\
3 & $0.674 \mathrm{D}+03$ & $0.256 \mathrm{D}-01$ \\
4 & $-0.103 \mathrm{D}+04$ & $-0.328 \mathrm{D}-02$ \\
5 & $0.148 \mathrm{D}+04$ & $0.399 \mathrm{D}-03$ \\
6 & $-0.203 \mathrm{D}+04$ & $-0.191 \mathrm{D}-04$ \\
7 & $0.269 \mathrm{D}+04$ & $-0.523 \mathrm{D}-05$ \\
8 & $-0.346 \mathrm{D}+04$ & $0.177 \mathrm{D}-05$ \\
9 & $0.435 \mathrm{D}+04$ & $-0.341 \mathrm{D}-06$ \\
10 & $-0.537 \mathrm{D}+04$ & $0.549 \mathrm{D}-07$ \\
11 & $0.651 \mathrm{D}+04$ & $-0.865 \mathrm{D}-08$ \\
12 & $-0.780 \mathrm{D}+04$ & $0.149 \mathrm{D}-08$ \\
13 & $0.922 \mathrm{D}+04$ & $-0.278 \mathrm{D}-09$ \\
14 & $-0.108 \mathrm{D}+05$ & $0.682 \mathrm{D}-10$ \\
15 & $0.125 \mathrm{D}+05$ & $-0.319 \mathrm{D}-11$ \\
16 & $-0.144 \mathrm{D}+05$ & $0.138 \mathrm{D}-10$ \\
17 & $0.164 \mathrm{D}+05$ & $0.115 \mathrm{D}-10$ \\
18 & $-0.186 \mathrm{D}+05$ & $0.136 \mathrm{D}-10$ \\
19 & $0.210 \mathrm{D}+05$ & $0.149 \mathrm{D}-10$ \\
\hline
\end{tabular}

as $n \rightarrow \infty$. If $\max _{0 \leq i \leq n+1}\left|F\left(x_{j+i}\right)\right|$ is of the order of $10^{\alpha}$, and if it has been computed to machine accuracy, e.g., to $p$ decimal digits, then $\max _{0 \leq i \leq n+1}\left|\varepsilon_{j+i}\right|$ is of the order of $10^{-p+\alpha}$. If, furthermore, $I[f]$ is of the order of $10^{\beta}$, then the relative error $|E / I[f]|$ will be of the order of $10^{-p+\alpha-\beta}$, i.e., the number of correct significant figures in the computed value of $W_{n}^{(j)}$ will be approximately $p+\beta-\alpha$. Finally, if $\beta<\alpha$, as is the case in Examples 2.1, 2.2, and 2.5, then this number is less than $p$. This suggests that, in case the sequence $F\left(x_{l}\right), l=0,1, \ldots$, is not bounded, which is the case for integrals with integrands in $\mathbf{B}_{d}$, then after a certain point the accuracy of the computed values of $W_{n}^{(j)}$ will diminish with increasing $n$.

Finally, we note that we can also take

$$
\psi\left(x_{l}\right)=\int_{x_{l-1}}^{x_{l}} f(t) d t, \quad l=0,1,2, \ldots, x_{-1}=a .
$$

The advantage of this choice is that $W_{n}^{(j)}$ is now determined by $j+n+2$ integrals $\psi\left(x_{0}\right), \ldots, \psi\left(x_{j+n+1}\right)$, one less than required by the choice of $\psi\left(x_{l}\right)$ given in (2.1). In many cases, however, the new choice $\left(2.1^{\prime}\right)$ results in less accuracy for the first few $W_{n}^{(0)}, n=0,1,2, \ldots$ Eventually, however, the convergence rate improves quickly.

3. Convergence Analysis. As is mentioned in Section 1, a sufficient condition for the convergence results in (1.18) and (1.19) is (1.16), which in turn is satisfied, since (1.17) holds for the original $W$-transformation. For the modified $W$-transformation, however, (1.16) need not hold in general. Nevertheless, as will be shown in Lemmas 3.3 and 3.8, for the modified $W$-transformation weaker forms of (1.16) and (1.17) exist, and these can be used to prove that (1.18) and (1.19) 
hold in some cases. Without making direct use of these weaker forms of (1.16) and (1.17), we shall prove that (1.18) (in fact, a much stronger version of it) and (1.19) hold in any case. It is important to note that these weaker forms are sufficient to guarantee the stability of the approximations $W_{n}^{(j)}$, both for Process I and Process II. The main results for Process I and Process II are given in Theorem 3.1 and Theorem 3.2, respectively.

Before we begin the analyses of convergence for $W_{n}^{(j)}$, we give closed-form expressions for $W_{n}^{(j)}$ and $I[f]-W_{n}^{(j)}$. In addition, we provide a thorough analysis of $\psi\left(x_{l}\right)$ and $x_{l}$ as functions of $l$. Throughout, we assume that $\psi\left(x_{l}\right) \neq 0, l=0,1, \ldots$

LEMMA 3.1. Let $\xi=1 / x, \xi_{i}=1 / x_{i}, i=0,1, \ldots$ Define $G(\xi) \equiv F(x)$, $\rho(\xi) \equiv \psi(x)$, and let $D_{n}^{(j)}$ and $E_{n}^{(j)}$ denote the divided difference operators over the sets of points $\left\{\xi_{j}, \xi_{j+1}, \ldots, \xi_{j+n+1}\right\}$ and $\left\{x_{j}, x_{j+1}, \ldots, x_{j+n+1}\right\}$, respectively. Then

$$
W_{n}^{(j)}=\frac{D_{n}^{(j)}\{G(\xi) / \rho(\xi)\}}{D_{n}^{(j)}\{1 / \rho(\xi)\}}
$$

and also

$$
W_{n}^{(j)}=\frac{E_{n}^{(j)}\left\{x^{n} F(x) / \psi(x)\right\}}{E_{n}^{(j)}\left\{x^{n} / \psi(x)\right\}} .
$$

Proof. The proof of (3.1) has been given in [5]. The proof of (3.2) can be accomplished by multiplying (1.9) on both sides by $x_{l}^{n}$, and then using the technique by which (3.1) is obtained.

Note that the $W$-algorithm described in (1.12) and (1.13) of the present work is based on (3.1). Equation (3.2), however, is new.

LEMMA 3.2. The error in $W_{n}^{(j)}$ can be expressed as

$$
I[f]-W_{n}^{(j)}=\frac{D_{n}^{(j)}\{(I[f]-G(\xi)) / \rho(\xi)\}}{D_{n}^{(j)}\{1 / \rho(\xi)\}}
$$

and also as

$$
I[f]-W_{n}^{(j)}=\frac{E_{n}^{(j)}\left\{x^{n}(I[f]-F(x)) / \psi(x)\right\}}{E_{n}^{(j)}\left\{x^{n} / \psi(x)\right\}} .
$$

Proof. (3.3) and (3.4) follow from Lemma 3.1 and the linearity of the operators $D_{n}^{(j)}$ and $E_{n}^{(j)}$.

LEMMA 3.3. For the modified $W$-transformation a weak form of (1.17), namely

$$
\psi\left(x_{l}\right) \psi\left(x_{l+1}\right)<0, \quad l \geq L, \text { some integer } L \geq 0,
$$

is valid. Consequently, a weak form of (1.16), namely

$$
\gamma_{n, i}^{(j)}>0, \quad 0 \leq i \leq n+1, j \geq L, \text { all } n,
$$

holds.

Proof. By (2.1) and (1.11),

$$
\psi\left(x_{l}\right)=(-1)^{l}\left[x_{l}^{\sigma+\gamma} \exp \left(\bar{\phi}\left(x_{l}\right)\right) b_{1}\left(x_{l}\right)+x_{l+1}^{\sigma+\gamma} \exp \left(\bar{\phi}\left(x_{l+1}\right)\right) b_{1}\left(x_{l+1}\right)\right] .
$$


Since $b_{1} \in A^{(0)}, b_{1}(x)$ is of one sign for all sufficiently large $x$. This, combined with the fact that $\lim _{l \rightarrow \infty} x_{l}=\infty$, implies that there exists a nonnegative integer $L$ for which $b_{1}\left(x_{i}\right), i \geq L$, are all of the same sign. Consequently, the expression inside the square brackets on the right-hand side of (3.7) is of the same sign for all $l \geq L$. The result now follows.

Throughout the remainder of this section, $T(l)$ will denote generically any function of $l$ that has a convergent expansion of the form $\sum_{i=0}^{\infty} T_{i} l^{-i / m}$ for all sufficiently large $l$. Note that since $l$ is equal to a polynomial in $x_{l}$ of degree exactly $m, T(l)$ is equal to a (generic) function $\hat{T}\left(x_{l}\right)$ that has a convergent expansion of the form $\sum_{i=0}^{\infty} \hat{T}_{i} x_{l}^{-i}$ for all sufficiently large $l$ (or $x_{l}$ ). Similarly, $U(l)$ will denote generically any function of $l$ that has an asymptotic expansion of the form $\sum_{i=0}^{\infty} U_{i} l^{-i / m}$ as $l \rightarrow \infty$. Again, $U(l)$ is equal to a (generic) function $\hat{U}\left(x_{l}\right)$ that has an asymptotic expansion of the form $\sum_{i=0}^{\infty} \hat{U}_{i} x_{l}^{-i}$ as $l$ (or $\left.x_{l}\right) \rightarrow \infty$. Needless to say, $\hat{T}(x)$ is in $A^{(0)}$. All the functions $\hat{U}(x)$ that we encounter in the remainder of this section can also be shown to be in $A^{(0)}$, although we omit the proofs.

LEMMA 3.4. As a function of $l$, and for all sufficiently large $l$,

(a) $x_{l}$ has the convergent expansion

$$
x_{l}=l^{1 / m} \sum_{i=0}^{\infty} \delta_{i} l^{-i / m}, \quad \delta_{0}>0 ;
$$

(b) $x_{l+1}$ has the convergent expansion

$$
x_{l+1}=l^{1 / m} \sum_{i=0}^{\infty} \tilde{\delta}_{i} l^{-i / m},
$$

where

$$
\tilde{\delta}_{i}=\delta_{i}, \quad 0 \leq i \leq m-1, \quad \tilde{\delta}_{m}=\delta_{m}+\delta_{0} / m
$$

(c) for any nonzero $p$,

$$
x_{l+1}^{p}-x_{l}^{p}=l^{(p-m) / m}\left[\frac{p \delta_{0}^{p}}{m}+l^{-1 / m} T(l)\right] .
$$

Proof. Since $x_{l}$ is the largest positive solution of the polynomial equation $\bar{\theta}(x)-$ $(q+l) \pi=0$, we can easily see that $x_{l}=O\left(l^{1 / m}\right)$ as $l \rightarrow \infty$. If we now let $\varepsilon=l^{-1 / m}$ and $x_{l}=\varepsilon^{-1} y(\varepsilon)$, and write $\bar{\theta}(x)=\sum_{j=0}^{m-1} \mu_{j} x^{m-j}$, then the polynomial equation above becomes $\sum_{j=0}^{m-1} \mu_{j} \varepsilon^{j} y^{m-j}-\left(1+q \varepsilon^{m}\right) \pi=0$. Note that the coefficient of $y^{m}$ is independent of $\varepsilon$, and the rest of the coefficients are polynomials in $\varepsilon$. Therefore, $y(\varepsilon)$ is an analytic function of $\varepsilon$ at $\varepsilon=0$. Consequently, for all $\varepsilon$ sufficiently close to zero, a convergent expansion of the form $y(\varepsilon)=\sum_{i=0}^{\infty} \delta_{i} \varepsilon^{i}$ exists. The $\delta_{i}$ can be determined by substituting this expansion for $y(\varepsilon)$ in the polynomial equation and equating the coefficients of each $\varepsilon^{i}$ to zero, and solving the resulting equations for $\delta_{0}, \delta_{1}, \delta_{2}, \ldots$, in this order. Actually, $\delta_{0}=\left(\pi / \mu_{0}\right)^{1 / m}>0$, since $\mu_{0}>0$ by assumption. This proves part (a). Part (b) follows directly from part (a) by replacing $l$ by $l+1$ in (3.8).

To prove part (c), we first observe that

$$
\frac{x_{l+1}}{x_{l}}=\frac{x_{l+1}-x_{l}}{x_{l}}+1=1+l^{-1}\left[\frac{1}{m}+l^{-1 / m} T(l)\right]
$$


the second equality following from (3.8)-(3.10). Raising both sides of the equality in (3.12) to the power $p$, we obtain

$$
\left(\frac{x_{l+1}}{x_{l}}\right)^{p}=1+l^{-1}\left[\frac{p}{m}+l^{-1 / m} T(l)\right],
$$

which in turn results in (3.11).

LEMMA 3.5. As a function of $x_{l}$,

$$
\psi\left(x_{l}\right)=(-1)^{l} x_{l}^{\sigma+\gamma} \exp \left(\bar{\phi}\left(x_{l}\right)\right) b_{1}\left(x_{l}\right)\left[1+\omega\left(x_{l}\right)\right]
$$

where

$$
\omega(x) \sim \sum_{i=0}^{\infty} \omega_{i} / x^{i} \quad \text { as } x \rightarrow \infty, \quad \omega_{0}>0, \quad \text { if } k \leq m,
$$

and $\omega \in A^{(0)}$, and

$$
\omega(x)=e^{P(x)} \hat{U}(x), \quad \lim _{x \rightarrow \infty} P(x)=-\infty, \quad \text { if } k>m,
$$

where $P(x)$ is a polynomial in $x$ of degree $k-m$ whose construction is explained through (3.18) (3.21) in the proof below.

Proof. (3.14) follows from (3.7), once we identify

$$
\omega\left(x_{l}\right)=\left(\frac{x_{l+1}}{x_{l}}\right)^{\sigma+\gamma} \frac{b_{1}\left(x_{l+1}\right)}{b_{1}\left(x_{l}\right)} \exp \left[\bar{\phi}\left(x_{l+1}\right)-\bar{\phi}\left(x_{l}\right)\right] .
$$

By Lemma 3.4, after some tedious manipulations, it can be shown that

$$
\left(\frac{x_{l+1}}{x_{l}}\right)^{\sigma+\gamma} \frac{b_{1}\left(x_{l+1}\right)}{b_{1}\left(x_{l}\right)}=1+l^{-1} U(l) \text {. }
$$

If $k=0(<m), \exp \left[\bar{\phi}\left(x_{l+1}\right)-\bar{\phi}\left(x_{l}\right)\right]=1$, thus $\omega\left(x_{l}\right)=2+l^{-1} U(l)$ for this case. By the remark preceding Lemma 3.4, (3.15a) now follows. If $k \geq 1$, writing $\bar{\phi}(x)=\sum_{i=0}^{k-1} \nu_{i} x^{k-i}, \nu_{0}<0$, and invoking Lemma 3.4, it follows that

$$
\bar{\phi}\left(x_{l+1}\right)-\bar{\phi}\left(x_{l}\right)=l^{(k-m) / m} \sum_{i=0}^{\infty} d_{i} l^{-i / m}, \quad d_{0}=\nu_{0} \delta_{0}^{k} / m<0,
$$

for all sufficiently large $l$. Again by the remark preceding Lemma 3.4, (3.18) can be expressed as

$$
\bar{\phi}\left(x_{l+1}\right)-\bar{\phi}\left(x_{l}\right)=x_{l}^{k-m} \sum_{i=0}^{\infty} \hat{d}_{i} x_{l}^{-i}, \quad \hat{d}_{0}<0,
$$

for all sufficiently large $l$. Now if $k \leq m$, then $\bar{\phi}\left(x_{l+1}\right)-\bar{\phi}\left(x_{l}\right)=O\left(x_{l}^{k-m}\right)=O(1)$ as $l \rightarrow \infty$, hence $\omega(x)$ is as in (3.15a). If $k>m$, however, (3.19) can be expressed in the form

$$
\bar{\phi}\left(x_{l+1}\right)-\bar{\phi}\left(x_{l}\right)=P\left(x_{l}\right)+Q\left(x_{l}\right)
$$

where

$$
P(x)=\sum_{i=0}^{k-m-1} \hat{d}_{i} x^{k-m-i}, \quad Q(x)=\sum_{i=k-m}^{\infty} \hat{d}_{i} x^{k-m-i}
$$


Obviously, $P(x)$ is a polynomial in $x$ of degree $k-m$ with leading coefficient negative, and $Q(x)=O(1)$ as $x \rightarrow \infty$. Combining all the above in (3.16) yields (3.15b).

Note that, in any case, $1+\omega(x) \sim K$ as $x \rightarrow \infty$, where $K$ is a fixed positive constant.

3.1. Analysis of Process I.

LEMMA 3.6. Using the notation of Lemma 3.1, we have that

$$
\left|E_{n}^{(j)}\left\{x^{n} / \psi(x)\right\}\right| \sim C_{1} \exp \left(-\bar{\phi}\left(x_{j+n+1}\right)\right) x_{j}^{m n-\sigma-\gamma} \text { as } j \rightarrow \infty,
$$

where $C_{1}$ is a positive constant independent of $j$.

Proof. From the theory of divided differences it is known that

$$
E_{n}^{(j)}\{g(x)\}=\sum_{s=0}^{n+1} c_{n, s}^{(j)} g\left(x_{j+s}\right)
$$

where

$$
c_{n, s}^{(j)}=\prod_{\substack{i=0 \\ i \neq s}}^{n+1}\left(x_{j+s}-x_{j+i}\right)^{-1}, \quad 0 \leq s \leq n+1 .
$$

Obviously, $c_{n, s}^{(j)} c_{n, s+1}^{(j)}<0,0 \leq s \leq n$. Since also $\psi\left(x_{j+s}\right) \psi\left(x_{j+s+1}\right)<0$ for $j \geq L$ by Lemma 3.3 , we can write

$$
\left|E_{n}^{(j)}\left\{x^{n} / \psi(x)\right\}\right|=\sum_{s=0}^{n+1}\left|c_{n, s}^{(j)}\right| x_{j+s}^{n} /\left|\psi\left(x_{j+s}\right)\right|, \quad j \geq L .
$$

Therefore, the asymptotic behavior of $\left|E_{n}^{(j)}\left\{x^{n} / \psi(x)\right\}\right|$ is determined as the sum of the asymptotic behaviors of the (positive) terms in the summation on the righthand side of (3.25). First,

$$
\left|c_{n, s}^{(j)}\right| \sim c_{n, s} x_{j}^{(m-1) n} \quad \text { as } j \rightarrow \infty
$$

where $c_{n, s}$ are some fixed positive constants. To show (3.26), we observe that

$$
x_{j+s}-x_{j+i}=\left(x_{j+s}-x_{j}\right)-\left(x_{j+i}-x_{j}\right)
$$

and that

$$
x_{j+s}-x_{j}=\sum_{i=1}^{s}\left(x_{j+i}-x_{j+i-1}\right) \sim\left(s \delta_{0} / m\right) j^{(1-m) / m} \quad \text { as } j \rightarrow \infty,
$$

by Lemma 3.4. Combining (3.27) and (3.28) in (3.24), and invoking the remark preceding Lemma $3.4,(3.26)$ follows. Substituting (3.14) and (3.26) in (3.25), we obtain

$$
\begin{array}{r}
\left|E_{n}^{(j)}\left\{x^{n} / \psi(x)\right\}\right| \sim \sum_{s=0}^{n+1} \frac{c_{n, s} x_{j}^{(m-1) n} x_{j+s}^{n}}{x_{j+s}^{\sigma+\gamma} \exp \left(\bar{\phi}\left(x_{j+s}\right)\right)\left|b_{1}\left(x_{j+s}\right)\right|\left[1+\omega\left(x_{j+s}\right)\right]} \\
\text { as } j \rightarrow \infty .
\end{array}
$$

Using now the facts that $x_{j+i} \sim x_{j}$ as $j \rightarrow \infty, b_{1} \in A^{(0)}$, and $1+\omega(x) \sim K$ as $j \rightarrow \infty$, where $K$ is a fixed positive constant, (3.29) can be expressed as

$$
\left|E_{n}^{(j)}\left\{x^{n} / \psi(x)\right\}\right| \sim\left(\max _{0 \leq i \leq n+1} \exp \left(-\bar{\phi}\left(x_{j+i}\right)\right)\right) C_{1} x_{j}^{m n-\sigma-\gamma} \text { as } j \rightarrow \infty .
$$

Finally using the fact that $\lim _{x \rightarrow \infty} \bar{\phi}(x)=-\infty,(3.22)$ follows from (3.30). 
LEMMA 3.7. Denote

$$
N_{n}^{(j)}=E_{n}^{(j)}\left\{x^{n}(I[f]-F(x)) / \psi(x)\right\} .
$$

Then, for some positive constant $C_{2}$,

$$
\left|N_{n}^{(j)}\right| \sim C_{2} x_{j}^{-\hat{n}-2} \quad \text { as } j \rightarrow \infty, \text { if } k \leq m \text {, some integer } \hat{n} \geq n,
$$

and

$$
N_{n}^{(j)}=O\left(x_{j}^{(n+1)(k-m)-1} e^{P\left(x_{j}\right)}\right) \quad \text { as } j \rightarrow \infty, \text { if } k>m,
$$

with $P(x)$ as given in Lemma 3.5 .

Proof. By (1.11) and (3.14),

$$
\frac{I[f]-F\left(x_{l}\right)}{\psi\left(x_{l}\right)}=\frac{1}{1+\omega\left(x_{l}\right)}, \quad l=0,1, \ldots
$$

Expanding now the right-hand side of (3.31) according to (3.23), and using (3.33), we obtain

$$
N_{n}^{(j)}=E_{n}^{(j)}\left\{\frac{x^{n}}{1+\omega(x)}\right\} .
$$

Consider now the case $k \leq m$. Then by $(3.15 \mathrm{a})$,

$$
\frac{x^{n}}{1+\omega(x)} \sim \sum_{i=0}^{\infty} \omega_{i}^{\prime} x^{n-i} \quad \text { as } x \rightarrow \infty .
$$

It is known that, for any function $g(x)$ that has $n+1$ continuous derivatives over $\left[x_{j}, x_{j+n+1}\right]$,

$$
E_{n}^{(j)}\{g(x)\}=\frac{1}{(n+1) !} g^{(n+1)}(\tilde{x}), \quad x_{j}<\tilde{x}<x_{j+n+1},
$$

as a consequence of which we also have

$$
E_{n}^{(j)}\left\{x^{i}\right\}=0, \quad 0 \leq i \leq n .
$$

Substituting (3.35) in (3.34), and using (3.37), we obtain

$$
N_{n}^{(j)} \sim E_{n}^{(j)}\left\{\sum_{i=n+1}^{\infty} \omega_{i}^{\prime} x^{n-i}\right\} \quad \text { as } j \rightarrow \infty .
$$

(3.32a) now follows by employing (3.36) and using the fact that $x_{j+i} \sim x_{j}$ as $j \rightarrow \infty$. Note that $\hat{n}=n$ if $\omega_{n+1}^{\prime} \neq 0$; otherwise $\hat{n}>n$.

Next, consider the case $k>m$. Then by $(3.15 \mathrm{~b})$,

$$
\frac{x^{n}}{1+\omega(x)}=\frac{x^{n}}{1+e^{P(x)} \hat{U}(x)}=x^{n} \sum_{s=0}^{\infty}(-1)^{s} e^{s P(x)}[\hat{U}(x)]^{s}
$$

for all sufficiently large $x$, since $\lim _{x \rightarrow \infty} P(x)=-\infty$. Thus, for all sufficiently large $j$,

$$
N_{n}^{(j)}=E_{n}^{(j)}\left\{\frac{x^{n}}{1+\omega(x)}\right\}=\sum_{s=0}^{\infty}(-1)^{s} E_{n}^{(j)}\left\{x^{n}[\hat{U}(x)]^{s} e^{s P(x)}\right\}
$$


The $s=0$ term vanishes by (3.37). The $s=1$ term is the dominant one in the rest of the summation. Hence (3.40) becomes

$$
N_{n}^{(j)} \sim-E_{n}^{(j)}\left\{x^{n} \hat{U}(x) e^{P(x)}\right\} \quad \text { as } j \rightarrow \infty .
$$

Invoking (3.36), after some lengthy manipulations, (3.32b) can be shown to hold.

We now state the main convergence result for Process I, in which $n$ is held fixed and $j \rightarrow \infty$.

THEOREM 3.1. As $j \rightarrow \infty$, the error in $W_{n}^{(j)}$ satisfies

$$
\left|I[f]-W_{n}^{(j)}\right| \sim \hat{C} x_{j}^{\sigma+\gamma-m n-\hat{n}-2} \exp \left(\bar{\phi}\left(x_{j+n+1}\right)\right) \quad \text { as } j \rightarrow \infty, \text { if } k \leq m,
$$

where $\hat{C}$ is a positive constant, and $\hat{n}$ is as in (3.32a), and

$$
\begin{array}{r}
I[f]-W_{n}^{(j)}=O\left(x_{j}^{\sigma+\gamma+(k-2 m) n+k-m-1} \exp \left(\bar{\phi}\left(x_{j+n+1}\right)+P\left(x_{j}\right)\right)\right) \\
\text { as } j \rightarrow \infty, \text { if } k>m .
\end{array}
$$

Proof. The proof is achieved by combining Lemmas 3.6 and 3.7 in (3.4). It should be mentioned that $(3.42 \mathrm{a})$ and $(3.42 \mathrm{~b})$ are made possible by the fact that (3.22) is an asymptotic equivalence.

Note that the result stated in Theorem 3.1 is better than that stated in (1.18) for all $k$ and $m$. First, when $k \leq m$, the right-hand side of $(3.42 \mathrm{a})$ is smaller than the right-hand side of $(1.18)$ by a factor of $x_{j}^{-m n-1} \exp \left(\bar{\phi}\left(x_{j+n+1}\right)-\bar{\phi}\left(x_{j}\right)\right)$ which tends to zero as $j \rightarrow \infty$ both for $k=0$ and $k>0$, since $\lim _{x \rightarrow \infty} \bar{\phi}(x)=-\infty$ and $x_{j+n+1}>x_{j}$. Next, when $k>m$, the right-hand side of $(3.42 \mathrm{~b})$; s smaller than the right-hand side of $(1.18)$ by a factor of $x_{j}^{(k-2 m+1) n+k-m} \exp \left(\bar{\phi}\left(x_{j+n+1}\right)-\bar{\phi}\left(x_{j}\right)+\right.$ $P\left(x_{j}\right)$ ) which also tends to zero as $j \rightarrow \infty$ because of $\lim _{x \rightarrow \infty} \bar{\phi}(x)=-\infty$ and $\lim _{x \rightarrow \infty} P(x)=-\infty$.

Comparing (3.42a) and (3.42b), one might conclude that $(3.42 \mathrm{~b})$ is inferior to (3.42a), since the power of $x_{j}$ in $(3.42 \mathrm{~b})$ is greater than that in $(3.42 \mathrm{a})$ by $S=$ $(k-m+1)(n+1)>0$ for $k>m, n \geq 0$. Actually, $(3.42 \mathrm{~b})$ is as good as (3.42a), the loss through $x_{j}^{S}$ being compensated for by the factor $\exp \left(P\left(x_{j}\right)\right)$, since, practically speaking, $\exp (P(x)) \approx \exp \left(d_{0} x^{k-m}\right)$ for $x \rightarrow \infty, d_{0}<0$ (see (3.18) and (3.21) in the proof of Lemma 3.5).

Finally, we mention that the result in (3.42a) holds for the original $W$-transformation, both when $k \leq m$ and $k>m$. The proof of this can be achieved exactly as that of $(3.42 \mathrm{a})$.

3.2. Analysis of Process II. The following lemma gives a weak form of (1.16) for the modified $W$-transformation in conjunction with Process II.

LEMMA 3.8. For fixed $j$,

$$
\lim _{n \rightarrow \infty} \gamma_{n, s}^{(j)}=0 \quad \text { for finite } s,
$$

and also

$$
\lim _{n \rightarrow \infty} \sum_{s=0}^{n+1}\left|\gamma_{n, s}^{(j)}\right|=1 .
$$

Proof. We begin by noting that

$$
\gamma_{n, s}^{(j)}=\frac{c_{n, s}^{(j)} / \rho\left(\xi_{j+s}\right)}{D_{n}^{(j)}\{1 / \rho(\xi)\}}
$$


where this time,

$$
c_{n, s}^{(j)}=\prod_{\substack{i=0 \\ i \neq s}}^{n+1}\left(\xi_{j+s}-\xi_{j+i}\right)^{-1}
$$

This can be shown by making use of (3.1) and (3.23) and (3.24), with $E_{n}^{(j)}$ in (3.23) and $x_{l}$ in $(3.24)$ replaced by $D_{n}^{(j)}$ and $\xi_{l}$, respectively.

Denote now $\delta_{p q}=\left|\xi_{j+p}-\xi_{j+q}\right|$ for all $p$ and $q$ (with $j$ fixed), and consider

$$
\Gamma_{n, s}^{(j)}=\left|\frac{\gamma_{n, s}^{(j)}}{\gamma_{n, n+1}^{(j)}}\right|=\prod_{\substack{i=0 \\ i \neq s}}^{n} \frac{\delta_{n+1, i}}{\delta_{s, i}}\left|\frac{\rho\left(\xi_{j+n+1}\right)}{\rho\left(\xi_{j+s}\right)}\right|
$$

Using $\rho\left(\xi_{i}\right)=\psi\left(x_{i}\right)$, Lemma 3.5, and Lemma 3.4, it follows that

$$
\rho\left(\xi_{j+n+1}\right) / \rho\left(\xi_{j+s}\right)=O\left(x_{n}^{\sigma+\gamma}\right)=O\left(n^{(\sigma+\gamma) / m}\right) \quad \text { as } n \rightarrow \infty .
$$

Since $\lim _{i \rightarrow \infty} \xi_{i}=0$, given $\varepsilon>0$, there exists a positive integer $M$ for which $\delta_{p q}<\varepsilon$ if $p, q>M$. Without loss of generality we take $\varepsilon<\delta_{s, s+1}$ and $M>s$. Also, since $\xi_{i}$ decreases monotonically, the sequence $\delta_{s, i}$ increases monotonically for $i \geq s+1$. Combining all this, we have for all $n \geq M$

$$
\prod_{\substack{i=0 \\ i \neq s}}^{n} \frac{\delta_{n+1, i}}{\delta_{s, i}}<\left(\prod_{\substack{i=0 \\ i \neq s}}^{M} \frac{\delta_{n+1, i}}{\delta_{s, i}}\right)\left(\frac{\varepsilon}{\delta_{s, s+1}}\right)^{n-M} .
$$

Combining (3.48) and (3.49) in (3.47), and letting $n \rightarrow \infty$, we obtain

$$
\lim _{n \rightarrow \infty} \Gamma_{n, s}^{(j)}=0
$$

By Lemma 3.3 and the fact that $c_{n, s}^{(j)} c_{n, s+1}^{(j)}<0,0 \leq s \leq n$, we have that $\gamma_{n, l}^{(j)}$ are of the same sign for $l \geq L-j$. This, combined with (3.50) and (1.15), is sufficient to prove (3.43) and $\gamma_{n, l}^{(j)}>0, l \geq L-j$, from which (3.44) follows easily. We omit the details.

Note that (3.43) is valid for the original $W$-transformation too, the proof of this being exactly as above. We leave the details to the reader.

THEOREM 3.2. As $n \rightarrow \infty$, the error in $W_{n}^{(j)}$ satisfies

$$
\left|I[f]-W_{n}^{(j)}\right| \leq\left(\sum_{s=0}^{n+1}\left|\gamma_{n, s}^{(j)}\right|\left|\psi\left(x_{j+s}\right)\right|\right) o\left(n^{-\mu}\right), \quad \text { any } \mu>0 .
$$

When $f \in \mathbf{B}_{c}$, i.e., $I[f]$ exists in the ordinary sense, the summation on the righthand side of (3.51) is bounded; otherwise, it grows at most like a fixed power of $n$. Consequently, in any case, (1.19) holds.

Proof. Consider the error expression given in (3.3). In analogy with the proof of Lemma 3.7 , this expression can be shown to be equivalent to

$$
I[f]-W_{n}^{(j)}=\frac{D_{n}^{(j)}\left\{1 /\left(1+\omega\left(\xi^{-1}\right)\right)\right\}}{D_{n}^{(j)}\{1 / \rho(\xi)\}} .
$$


Now it follows from Lemma 3.5 that the function $\tilde{\omega}(\xi)=1 /\left(1+\omega\left(\xi^{-1}\right)\right)$ is infinitely differentiable for $\xi \in\left[0, \xi_{0}\right]$. Let $\pi_{n}(\xi)$ be the best polynomial approximation of degree at most $n$ to $\tilde{\omega}(\xi)$ on $\left[0, \xi_{j}\right]$ in the Chebyshev norm. Using the fact that

$$
D_{n}^{(j)}\left\{\xi^{i}\right\}=0, \quad 0 \leq i \leq n,
$$

(3.52) can now be written as

$$
I[f]-W_{n}^{(j)}=\frac{D_{n}^{(j)}\left\{\tilde{\omega}(\xi)-\pi_{n}(\xi)\right\}}{D_{n}^{(j)}\{1 / \rho(\xi)\}}
$$

which, by $(3.45)$, can be expanded as

$$
I[f]-W_{n}^{(j)}=\sum_{s=0}^{n+1} \gamma_{n, s}^{(j)} \psi\left(x_{j+s}\right)\left(\tilde{\omega}\left(\xi_{j+s}\right)-\pi_{n}\left(\xi_{j+s}\right)\right) .
$$

By a standard result in the theory of polynomial approximation, the fact that $\tilde{\omega}(\xi)$ is infinitely differentiable on $\left[0, \xi_{j}\right]$ implies that

$$
\max _{0 \leq \xi \leq \xi_{j}}\left|\tilde{\omega}(\xi)-\pi_{n}(\xi)\right|=o\left(n^{-\mu}\right), \quad \text { as } n \rightarrow \infty, \text { any } \mu>0 .
$$

Using (3.56) in (3.55), (3.51) now follows. The rest of the theorem follows by noting

$$
\sum_{s=0}^{n+1}\left|\gamma_{n, s}^{(j)}\right|\left|\psi\left(x_{j+s}\right)\right| \leq\left(\sum_{s=0}^{n+1}\left|\gamma_{n, s}^{(j)}\right|\right) \max _{0 \leq s \leq n+1}\left|\psi\left(x_{j+s}\right)\right|,
$$

Lemma 3.5 , and (3.44).

Finally, we note that both for Process I and Process II, $W_{n}^{(j)}$ can easily be shown to be a regular summability method, in that all conditions of the SilvermanToeplitz theorem [2, pp. 23-27] are satisfied. This implies that if the sequence $F\left(x_{l}\right), l=0,1,2, \ldots$, converges, then so do the sequences $W_{n}^{(j)}, j=0,1,2, \ldots$, and $W_{n}^{(j)}, n=0,1,2, \ldots$ Of course, this approach to the convergence analysis is too simplistic and crude, since no information concerning rates of convergence is obtained. Furthermore, if the sequence $F\left(x_{l}\right), l=0,1,2, \ldots$, does not converge, which is the case when $I[f]$ is defined in the summability sense but not in the ordinary sense, then no information on the convergence of $W_{n}^{(j)}$ is obtained.

4. Concluding Remarks. In this work we have proposed and analyzed in detail a modification of the $W$-transformation. The $W$-transformation was designed for efficient evaluation of oscillatory infinite integrals with integrands $f(x)$ in the class $\mathbf{B}$ and involves some amount of asymptotic analysis of the integrand $f(x)$ for $x \rightarrow \infty$. In fact, both the phase of oscillations and the amplitude have to be analyzed so as to extract information that is used as input for the $W$-transformation. The modification that we have proposed for the $W$-transformation requires asymptotic knowledge of only the phase of oscillations and requires no information on the amplitude. Furthermore, it requires no extra computational work and is as effective as the original $W$-transformation. It can be implemented very efficiently by the $W$-algorithm.

As suggested by ample numerical evidence, this modification can also be successfully applied to oscillatory infinite integrals with integrands not necessarily in B. From this observation one might conclude that the choice of the $x_{l}$ is the crucial 
ingredient for the success of both the original and the modified $W$-transformations. It is important to note that for both methods the choice of the $x_{l}$ is also very simple, as it involves the determination of the largest positive zero of a polynomial of degree $m$. This problem has immediate solution when $m=1$ or 2 , and is a simple numerical problem for $m \geq 3$.

Acknowledgment. This work was supported in part by the Fund for the Promotion of Research at the Technion.

Computer Science Department

Technion-Israel Institute of Technology

Haifa 32000 , Israel

1. I. M. LONGMAN, "Note on a method for computing infinite integrals of oscillatory functions," Proc. Cambridge Philos. Soc., v. 52, 1956, pp. 764-768.

2. R. E. Powell \& S. M. ShaH, Summability Theory and its Applications, Van Nostrand Reinhold, London, 1972.

3. A. SIDI, "Extrapolation methods for oscillatory infinite integrals," J. Inst. Math. Appl., v. 26, 1980, pp. 1-20.

4. A. SIDI, "The numerical evaluation of very oscillatory infinite integrals by extrapolation," Math. Comp., v. 38, 1982, pp. 517-529.

5. A. SIDI, "An algorithm for a special case of a generalization of the Richardson extrapolation process," Numer. Math., v. 38, 1982, pp. 299-307.

6. A. SIDI, "Extrapolation methods for divergent oscillatory infinite integrals that are defined in the sense of summability," J. Comput. Appl. Math., v. 17, 1987, pp. 105-114. 